\title{
Evaluaciones Prácticas Objetivadas en Anatomía. Diferencias de Rendimiento en Preguntas Realizadas en Modelos, Preparaciones Anatómicas y Cadáveres
}

\author{
Objetive Structured Practical Test in Anatomy. Differences in the Score \\ of Questions Realized in Models, Anatomical Preparations and Cadavers
}

Oscar Inzunza H. \& Guillermo Salgado A.

INZUNZA, H. O. \& SALGADO, A. G. Evaluaciones prácticas objetivadas en anatomía. Diferencias de rendimiento en preguntas realizadas en modelos, preparaciones anatómicas y cadáveres. Int. J. Morphol., 29(2):490-495, 2011.

RESUMEN: En la mayoría de los cursos de anatomía de nuestros país los temidos exámenes prácticos se realizan ahora en el formato de pruebas prácticas objetivadas; actividades no menos temidas pero que si ofrecen una similitud de condiciones a todos los examinados. En nuestro caso y atendida la escasez de material cadavérico, una parte de las estaciones de evaluación ocupan preparaciones anatómicas parafinadas o plastinadas y modelos anatómicos de serie que existen en el mercado; material que por sus características no siempre reflejan, en forma fidedigna, la realidad anatómica. En este estudio se compara el rendimiento de los alumnos de nuestros cursos de anatomía en a) disecciones cadavéricas b) preparaciones anatómicas plastinadas; y, c) distintos tipos de modelos anatómicos fabricados especialmente para la docencia en anatomía. Los resultados obtenidos muestran distinto grado de dificultad según el elemento sobre el cual se realiza la evaluación. Así, las peguntas sobre modelos son respondidas con mayor facilidad (?=3,219; escala de 0 a 4 puntos) que aquellas realizadas sobre preparaciones $(?=3,193)$ o cadáveres $(?=2,537)$. Además, hemos detectado que algunos modelos ofrecen mayor dificultad que otros -sobre una misma región anatómica- en el reconocimiento de estructuras anatómicas vasculares. Considerando estos datos, parece necesario extremar los cuidados al planificar estos tipos de evaluaciones prácticas que mezclan modelos, preparaciones anatómicas y cadáveres, a fin de minimizar los factores que inducen algún grado de disparidad en la evaluación.

PALABRAS CLAVE: Educación; Anatomía; Modelos anatómicos; Evaluación.

\section{INTRODUCCIÓN}

En los últimos lustros la docencia para los cursos de anatomía ha debido enfrentar una realidad cambiante caracterizada por: $1^{\circ}$.- La reducción del creditaje de los cursos (Drake et al., 2002); $2^{\circ}$.- El menguado acceso al material cadavérico, situación paradojal en nuestro medio ya que si se aplicara la legislación nacional vigente, todos los Departamentos de Anatomía podrían contar con suficiente material anatómico según consta en el Libro $9^{\circ}$ del Código Sanitario (2011); $3^{\circ}$.- La reducción notable de los cultores de las ciencias morfológicas (Inzunza et al., 2007), probablemente debido a la falta de expectativas de desarrollo y de financiamiento de las investigaciones que abordan aspectos macroscópicos de esta disciplina; $4^{\circ}$.- El incremento alarmante de número de alumnos por curso, situación que es extrema en las carreras de Enfermería y Obstetricia; hecho que altera peligrosamente la relación docente-alumno (Índices: Estadís- ticas y bases de datos del cned, 2011). Esta desmedrada situación ha forzado a los anatomistas a enfrentar de una manera distinta los temas morfológicos. Así, en la mayoría de los cursos se ha reemplazado la disección tradicional por la prospección de material cadavérico guiada por un docente. De igual forma, se ha incrementado el uso de preparaciones anatómicas tratadas con diversos métodos de conservación como parafinado o plastinado. Del mismo modo, se están utilizando cada vez más fantomas y modelos anatómicos, de alto costo, que pretenden imitar (no siempre con éxito) preparaciones cadavéricas.

Para ser consecuentes con los recursos metodológicos utilizados, necesariamente las evaluaciones prácticas de anatomía han debido afrontar notables cambios estructurales. Así, los temidos exámenes prácticos de anatomía se realizan aho- 
ra en el formato de pruebas prácticas objetivadas, homólogos de las evaluaciones clínicas de objetivos estructurados (ECOE) (Tapia et al., 2008); actividades no menos temidas pero que sí ofrecen una equidad en los temas que el alumno debe resolver y en los tiempos que el estudiante dispone para este efecto. Estas evaluaciones permiten observar en los alumnos no sólo habilidades prácticas básicas como la identificación de estructuras sino, también, capacidades de razonamiento y resolución de problemas en función de determinadas claves morfológicas que se presentan durante el desarrollo de la evaluación.

En este contexto, este trabajo analiza el rendimiento de los alumnos de la carrera de Enfermería en las evaluaciones prácticas de reconocimiento de estructuras anatómicas utilizando diversos recursos docentes, tales como: cadáveres, preparaciones cadavéricas y modelos anatómicos.

\section{MATERIAL Y MÉTODO}

En este trabajo se analizan los resultados obtenidos por 120 alumnos, en una de las evaluaciones prácticas del curso de Anatomía para la carrera de Enfermería de la Facultad de Medicina de la Pontificia Universidad Católica de Chile. En estas evaluaciones, diseñadas de manera similar a una prueba tipo ECOE, pretendemos ofrecer a los alumnos condiciones similares en el nivel de dificultad y en el tiempo de respuesta para cada pregunta. En estas pruebas (coloquialmente llamadas "gymkanas"), con el fin de optimizar el espacio físico de nuestro Pabellón de Anatomía y de reducir el tiempo total de ellas se forman dos filas (A y
B). Cada una de ellas cuenta con un total de 15 estaciones donde se utiliza material anatómico (cadáver, preparación anatómica tratada o modelo) y material computacional dispuesto en cuatro computadores con fotos de preparaciones anatómicas tratadas como animaciones (Inzunza \& Bravo, 2002). En cada estación los alumnos deben identificar cuatro elementos anatómicos en 100 segundos. De estas estaciones, diez son segregadas (teóricamente iguales para cada grupo) y las otras cinco compartidas entre ambos grupos; en estas últimas, los alumnos de una fila abordan el material anatómico por el lado derecho mientras que los de la otra lo hace por el lado izquierdo. En cada fila los alumnos van pasando sucesivamente por cada estación. Con esta planificación, el tiempo total de la prueba práctica es de 1,8 horas y, para cada alumno el tiempo real de evaluación es de 25 minutos; si hiciéramos una fila única, el tiempo total de la prueba ascendería a 3,25 horas y el último alumno debería esperar casi tres horas para ingresar al Pabellón de Anatomía a realizar su examen.

Se comparan los puntajes obtenidos por los alumnos en la prueba práctica de reconocimiento de estructuras anatómicas del capítulo de Aparato Locomotor y Aparato Circulatorio. Las mismas preguntas, referidas a identificar estructuras anatómicas, fueron realizadas a cada mitad de curso (fila A v/s fila B) con diferencias en: a) los recursos docentes utilizados, b) el grado de disección de los cadáveres y, c) el lado por cual abordaron el material cadavérico. Se analizaron comparativamente los promedios de puntos obtenidos por cada mitad de curso en cada una de las preguntas analizadas. En la Tabla I se detallan las preguntas investigadas, señalando las características de los recursos docentes utilizados para cada mitad del curso.

Tabla I. Preguntas comparativas de reconocimiento de estructuras anatómicas.

- MOD 3A: Pregunta de reconocimiento de estructuras anatómicas en un modelo anatómico de 3B Scientific $®$.

- MOD 5A: Pregunta de reconocimiento de estructuras anatómicas en un modelo anatómico de 3B Scientific ${ }^{\circledR}$ (Fig. 1a).

- PREP 6A: Pregunta de reconocimiento de estructuras anatómicas en una preparación anatómica plastinada.
- PREP 3B: Pregunta de reconocimiento de estructuras anatómicas en una preparación anatómica conservada en líquido fijador.

- PREP 5B: Pregunta de reconocimiento de estructuras anatómicas en una preparación anatómica parafinada (Fig. 1b).

- MOD 6B: Pregunta de reconocimiento de estructuras anatómicas en un modelo anatómico de 3B Scientific®.

- MOD-D 11A: Pregunta de reconocimiento de estructuras anatómicas en un modelo anatómico de Bobbitt Laboratories ${ }^{\circledR}$, con alto grado de dificultad (Fig. 2a).

- MOD-F 11B: Pregunta de reconocimiento de estructuras anatómicas en un modelo anatómico de Denoyer-Geppert®, con bajo grado de dificultad (Fig. 2b).

- CAD-F 13A: Pregunta de reconocimiento de estructuras - CAD-D 13B: Pregunta de reconocimiento de estructuras anatómicas en un cadáver disecado y c onocido previamente por los alumnos (Fig. 3a). anatómicas en un cadáver disecado al efecto, no conocido previamente por los alumnos (Fig. 3b).

- CAD-AD 14A: Pregunta de reconocimiento de estructuras anatómicas en un cadáver, abordándolo por el lado derecho.
- CAD-AI 14B: Pregunta de reconocimiento de estructuras anatómicas en un cadáver, abordándolo por el lado izquierdo. 


\section{RESULTADOS}

Los resultados obtenidos en las distintas modalidades de preguntas aparecen destacados en la Tabla II. La escala de puntos utilizada fue de 0 (cero) a 4 (cuatro). Aquí se aprecia, en general, una gradiente de rendimiento que es mayor en las preguntas sobre modelos y menor en las preguntas sobre cadáveres (Figs. 1 a 4). Este dato se ve refrendado cuando se compara la sumatoria del puntaje obtenido en modelos, preparaciones y cadáveres (Tabla III). Al aplicar el análisis de varianza y la comparación múltiple de pares con el método de Dunn, se aprecia que con excepción de la pregunta 3 las diferencias observadas son significativas (Tabla IV).

Tabla II. Promedio de puntos obtenidos, en cada pregunta, en las filas A y B.

\begin{tabular}{|c|c|c|c|}
\hline Pregunta & $\begin{array}{c}\text { Puntos } \\
\text { promedio }\end{array}$ & SD & Significancia \\
\hline Modelo $3 A$ & 2,745 & 0,65 & \multirow{2}{*}{ NO } \\
\hline Preparación $3 \boldsymbol{B}$ & 2,667 & 0,82 & \\
\hline Modelo $5 A$ & 3,520 & 0,78 & \multirow{2}{*}{ SI } \\
\hline Preparación $5 \boldsymbol{B}$ & 3,255 & 0,97 & \\
\hline Preparación $6 A$ & 3,657 & 0,79 & \multirow{2}{*}{ SI } \\
\hline Modelo $6 \boldsymbol{B}$ & 3,392 & 0,94 & \\
\hline Modelo fácil $11 \boldsymbol{B}$ & 3,539 & $0, \hat{99}$ & \multirow{2}{*}{ SI } \\
\hline Modelo difícil $11 A$ & 3,265 & 1,15 & \\
\hline Cadáver fácil $13 A$ & 2,412 & 1,32 & \multirow{2}{*}{ SI } \\
\hline Cadáver difícil $13 B$ & 2,137 & 1,24 & \\
\hline Cadáver abordaje por derecha $14 A$ & 3,000 & 1,14 & \multirow[b]{2}{*}{ SI } \\
\hline Cadáver abordaje por izquierda $14 \boldsymbol{B}$ & 2,598 & 1,47 & \\
\hline
\end{tabular}

* Comparación múltiple de pares, método de Dunn. p < 0,05

En relación con las preguntas sobre vasos ilíacos realizadas en dos tipos de modelos (Bobbitt Laboratories ${ }^{\circledR}$ v/s Denoyer-Geppert ${ }^{\circledR}$ ) se observan las diferencias significativas de puntaje, siendo más complejo de reconocer el modelo de Bobbitt Laboratories ${ }^{\circledR}$; modelo que muestra la región pélvica con parte del peritoneo de la zona. Por su parte, el modelo de Denoyer-Geppert ${ }^{\circledR}$ presenta esta región totalmente desprovista de esa serosa (Tabla II y Fig. 2).
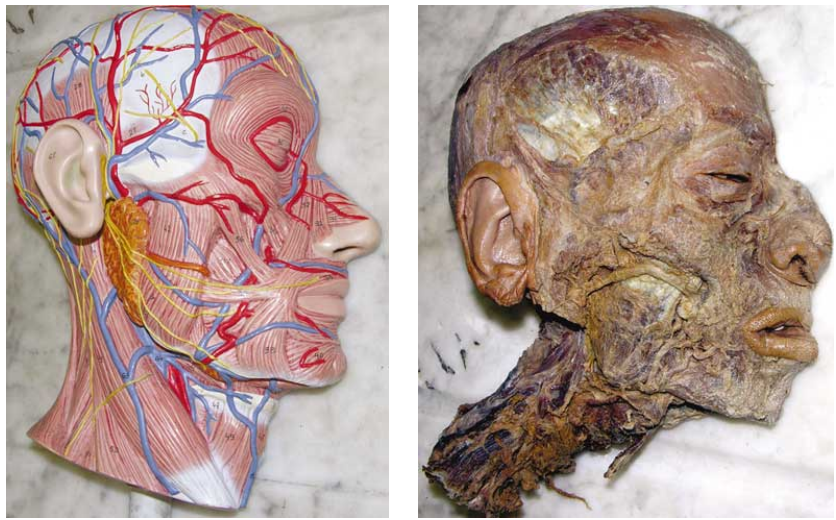

Tabla III. Sumatoria de los resultados en modelos, preparaciones y cadáveres.

\begin{tabular}{lcc}
\hline Recurso & Prome dio & SD \\
\hline Modelos & 3,219 & 0,866 \\
Preparaciones & 3,193 & 0,953 \\
Cadáveres & 2,537 & 1,332 \\
\hline
\end{tabular}

Tabla IV. Sumatoria del rendimiento en modelos, preparaciones y cadáveres. Comparación múltiple de pares, método de Dunn.

\begin{tabular}{lc}
\hline Recurso & Significancia \\
\hline Modelos v/s Preparaciones & NO \\
Modelos v/s Cadáveres & SI \\
Preparaciones v/s Cadáveres & SI \\
\hline $\mathrm{p}<0,05$ &
\end{tabular}

Tabla V. Promedio de notas finales en la evaluación práctica.

\begin{tabular}{lcc}
\hline Fila & Promedio & SD \\
\hline Notas Fila $\boldsymbol{A}$ & 5,400 & 0,734 \\
Notas Fila $\boldsymbol{B}$ & 5,219 & 0,961 \\
\hline
\end{tabular}

Respecto de las preguntas sobre cadáveres, las disecciones realizadas al efecto para una evaluación representan un complejidad mayor para estos alumnos; aún cuando esa región anatómica en particular fue revisada ampliamente en la antímera contralateral (Tabla II y Fig. 3).

Así mismo, en relación con las preguntas realizadas en cadáveres de estructuras anatómicas dispuestas en el plano sagital, como es el caso de las ramas viscerales impares de la aorta abdominal, los alumnos que abordan la zona por el lado derecho presentan un rendimiento mayor que aquellos que lo hacen por la antímera opuesta (Tabla II y Fig. 4).
Fig. 1. a) Modelo anatómico de cara y cuello, de la firma 3B Scientific $\AA$, que muestra la disposición de los músculos faciales y masticadores. b) Preparación anatómica de cara y cuello que muestra la disposición de los músculos faciales y masticadores; material mantenido en líquido fijador. 

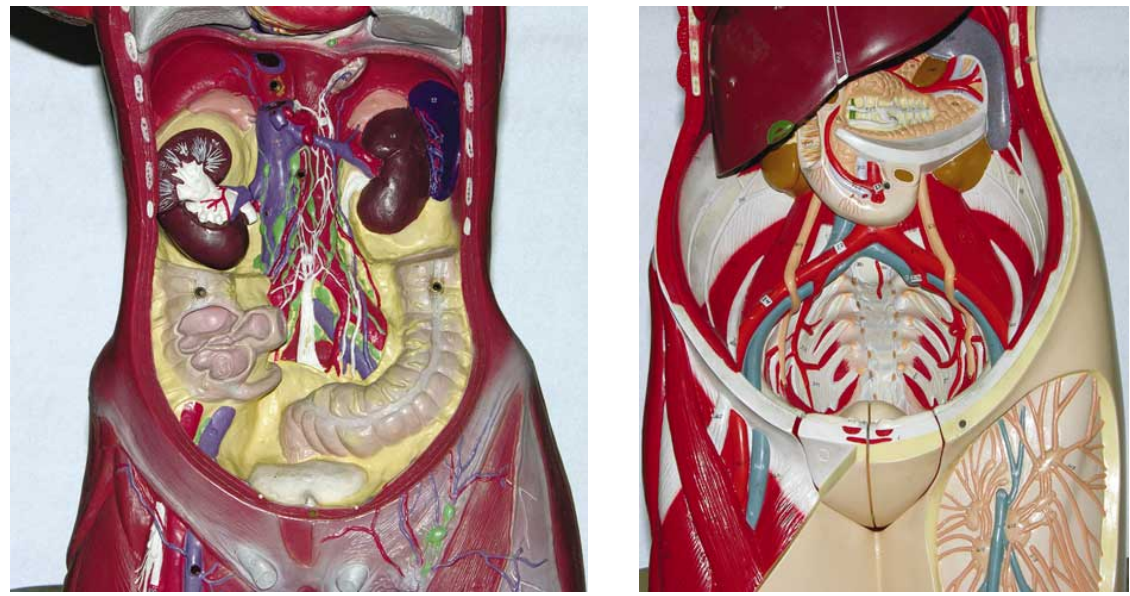

Fig. 2. a) Modelo de la firma Bobbitt Laboratories ${ }^{\circledR}$, que muestra la anatomía del tronco. Esta maqueta muestra las vísceras de la región abdominopélvica con parte del peritoneo de la región. b) Modelo de la firma DenoyerGeppert®, que muestra la anatomía del tronco. Esta maqueta muestra la región abdomino-pélvica desprovista de peritoneo.
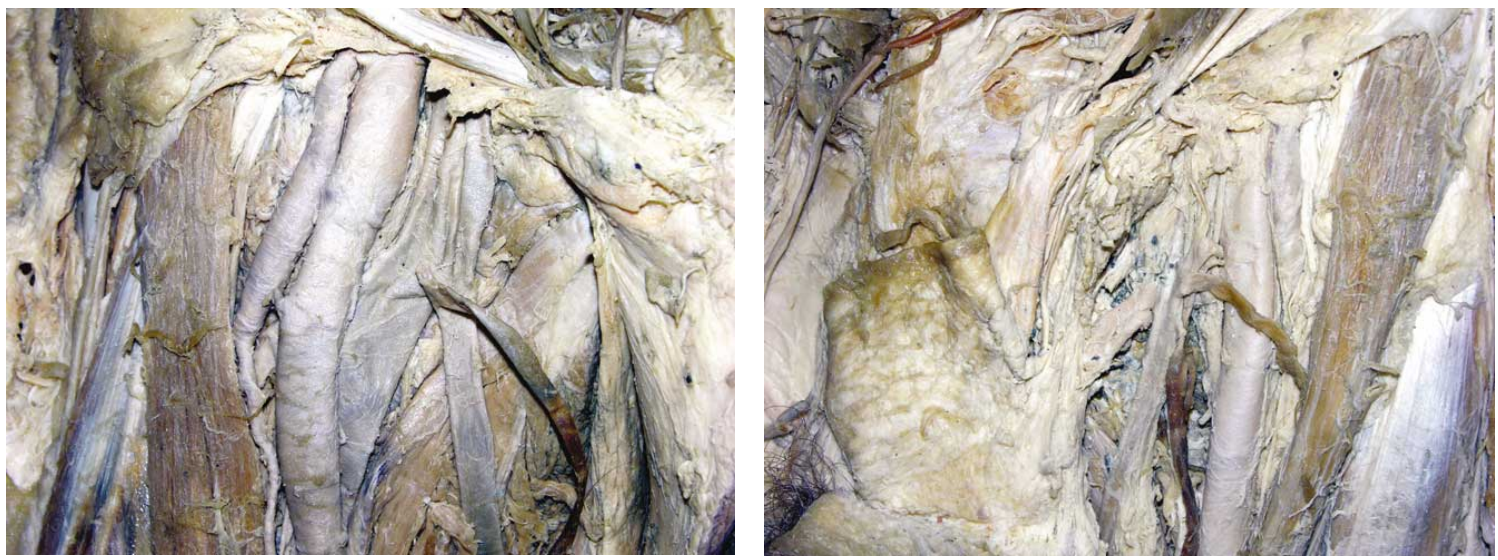

Fig. 3. a) Región del triángulo femoral de un cadáver utilizado en los pasos prácticos del curso, cuyos elementos fueron requeridos a los alumnos de la fila A. b) Región del triángulo femoral de un cadáver, cuyos elementos fueron disecados para efectos de la evaluación y requeridos a los alumnos de la fila B.

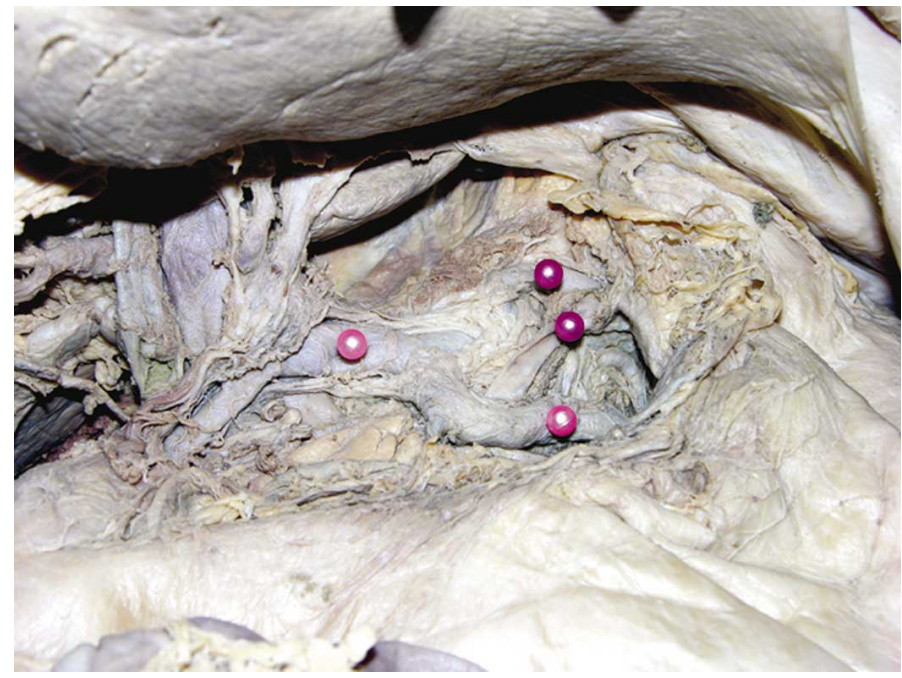

Fig. 4. Disección del tronco celíaco y sus ramas en un cadáver utilizado en los pasos prácticos del curso. Durante la evaluación práctica los alumnos de la fila A abordaron el cadáver por el lado derecho mientras que los de la fila B lo hicieron por el lado izquierdo.
Por último, sin perjuicio de los resultados obtenidos por estos alumnos en las distintas modalidades en que fueron requeridos en esta prueba práctica objetivada, no observamos diferencias significativas en la nota final entre las filas A y B (Tabla V). 


\section{DISCUSIÓN}

En los últimos años hemos asistido a un proceso continuo de cambios en la docencia de anatomía, caracterizados por: 1.- Un incremento en el número de alumnos por curso, situación que es extrema en las carreras de Enfermería y Obstetricia; 2.- La reducción notable del personal docente calificado que acompaña a estos cursos; 3.- La disminución del material cadavérico disponible para estos cursos; y, 4.- La aparición de numerosa oferta de modelos tridimensionales de apoyo a la docencia en anatomía (Inzunza \& Bravo).

Por otro lado, la Morfología en general y la Anatomía en particular corresponden a ciencias donde las claves visuales son extremadamente relevantes para su aprendizaje (Norvis, 1971; Bravo \& Inzunza, 1995). Además, estas asignaturas demandan de una manera novedosa el sensorio y la mente de nuestros alumnos quienes, acostumbrados a responder preguntas de alternativas, deben enfrentarse ahora a reconocer y relacionar elementos anatómicos en cadáveres, preparaciones anatómicas y modelos anatómicos tridimensionales (Inzunza et al., 2003).

El análisis de los resultados obtenidos muestra una gradiente, donde se observan mejores resultados en el reconocimiento de estructuras anatómicas en modelos y peores resultados en cadáveres (Tabla III). Esta situación es esperable ya que los modelos utilizados en esta evaluación tienen un grado de complejidad media y las preguntas sobre cadáveres tradicionalmente han representado un escollo mayor en todas las evaluaciones de Anatomía (Inzunza et al., 2003).

Sin perjuicio de lo anterior, existen modelos que son más esquemáticos, en los cuales es más fácil reconocer los distintos elementos anatómicos que presentan (Fig. 2a). En cambio, aquellos modelos que pretenden remedar una disección anatómica restringida de la región pélvica (Fig. 2b), constituyen un obstáculo mayor para la identificación de las estructuras anatómicas que ofrece. Las diferencias observadas en las respuestas de los alumnos en estos dos tipos de modelos son significativas; situación que introduce un factor de disparidad en la evaluación que es necesario considerar.

Del mismo modo, las disecciones realizadas al efecto para el proceso evaluativo son más difíciles de responder que las disecciones analizadas en los pasos prácticos por los alumnos, aún cuando correspondan exactamente a los elementos presentados en estas últimas. Por ejemplo, la disección de vasos del triángulo femoral en la estación 13 (Tabla II). Esta situación también la hemos observado en las evaluaciones realizadas a los alumnos de medicina (Inzunza et al., 2002), hecho que confirma la importancia de la claves visuales, en términos de situación, relaciones y topografía, para la identificación exitosa de los elementos de una preparación anatómica (Norvis; Inzunza \& Bravo).

El análisis de la pregunta 14 de nuestra prueba (Tabla II) muestra diferencias significativas en el rendimiento, entre los alumnos de la fila A y de la fila B, para identificar estructuras que se encuentran cercanas al plano sagital, como son por ejemplo las ramas viscerales impares de la aorta abdominal. En esta caso, llama la atención que la fila que aborda el cadáver por el lado derecho presenta un mejor rendimiento que aquellas que lo hace por el lado izquierdo; situación que también hemos observado en los cursos de medicina. Probablemente, esto sea una consecuencia del desarrollo de la actividad práctica de estos temas, donde el prosector realiza su demostración al lado derecho del cadáver con los alumnos siguiendo la presentación a los lados del docente. Otro factor posible a considerar en este punto es la insuficiente internalización, por parte de los alumnos, de la posición anatómica como base para la descripción en morfología.

Los datos aquí presentados muestran una situación paradojal ya que, al intentar ofrecer una evaluación práctica programada, estandarizada en términos del tiempo asignado para cada alumno y en igualdad de condiciones para todos los examinandos, surgen diferencias en el grado de dificultad entre las filas A y B; esto debido a la utilización de distintos elementos anatómicos como base para las preguntas de la prueba práctica (Tabla II). Afortunadamente, estas disparidades se logran alternar entre la filas, de manera que las diferencias en las notas finales de la evaluación práctica no son significativas (Tabla IV y Tabla V). Sin perjuicio de lo anterior, se deben extremar los cuidados en el proceso de generación de estas pruebas prácticas de reconocimiento de estructuras anatómicas sobre distintos recursos docentes ya sea cadáver, preparación anatómica o modelo anatómico; a fin de eliminar o reducir al mínimo posible estos factores perturbadores que inducen algún grado de disparidad en la evaluación. Aparentemente, así como la justicia es ciega nuestra evaluación también lo es.

INZUNZA, H. O. \& SALGADO, A. G. Objetive structured practical test in anatomy. Differences in the score of questions realized in models, anatomical preparations and cadavers. Int. J. Morphol., 29(2):490-495, 2011.

SUMMARY: In most anatomy courses in our country practical tests are now performed in objectified format, activities that provide similar conditions for all examinees. In our case and 
attending the shortage of cadaveric material, part of the evaluation stations use plastinated or paraffined anatomical preparations and serial anatomical models; material which by its nature, does not always reflect real anatomy in a trustworthy manner. This study compares the performance of students in our courses of anatomy in a) cadaveric dissections b) plastinated anatomical preparations, and c) different types of anatomical models made specifically for teaching anatomy. The results show varying degrees of difficulty depending on the element on which the assessment is realized. Questions made in models are more easily answered $(?=3.219$; scale of 0 to 4 points) than those performed on anatomical preparations $(?=3.193)$ or cadaver $(?=2.537)$. In addition, we found that some models offer greater difficulty than others of the same anatomical region. Considering these facts it seems necessary to be particularly careful during the planning of this type of practical test, which includes models, anatomical preparations and cadavers, in order to avoid those factors which induce some grade of disparity

KEY WORDS: Education; Anatomy; Anatomical models; Evaluation.

\section{REFERENCIAS BIBLIOGRÁFICAS}

Bravo, H. \& Inzunza, O. Evaluación de algunos programas computacionales en la enseñanza de la anatomía y neuroanatomía de la Facultad de Medicina de la Pontificia Universidad Católica de Chile. Rev. Chil. Anat., 13(1):7986, 1995.

Código Sanitario. Disponible en: http://epi.minsal.cl/, consultado el 18 de Abril del 2011

Drake, R. L.; Lowrie, D. J. \& Prewitt, Ch. M. Survey of gross anatomy, microscopic anatomy, neuroscience, and embryology courses in medical school curricula in the United States. Anat. Rec., 269(2):118-22, 2002.

Índices: Estadísticas y bases de datos del cned. Disponible en: http://www.cned.cl/public/Secciones/Seccion IndicesEstadisticas/indices_estadisticas_sistema.aspx, consultado el 18 de Abril del 2011.

Inzunza, O. \& Bravo, H. Animación computacional de fotografías, un real aporte al aprendizaje práctico de anatomía humana. Rev. Chil. Anat., 20(2):151-7, 2002.

Inzunza, O.; D'Acuña, E. \& Bravo, H. Evaluación práctica de Anatomía. Rendimiento de los alumnos de primer año de Medicina ante distintas formas de preguntar. Int. J. Morphol 21(2):131-6, 2003.

Inzunza, O.; Vargas, A. \& Bravo, H. Anatomía y Neuroanatomía, Disciplinas Perjudicadas por la Refor- ma Curricular. Int. J. Morphol., 25(4):825-30, 2007.

Norvis, G. Didáctica y estructura de los medios audiovisuales. Buenos Aires, Editorial Kapeluz, 1971.

Tapia, R.; Núñez, R.; Salas, R. \& Rodriguez-Orozco, A. El examen clínico objetivo estructurado como herramienta para la evaluación de competencias clínicas del internado de pregrado. Educ. Med. Super., 22(1):0-0, 2008.

\section{Dirección para correspondencia: \\ Prof. Dr. Oscar Inzunza \\ Departamento de Anatomía Normal \\ Escuela de Medicina \\ Pontificia Universidad Católica de Chile \\ CHILE}

Teléfono 56-2-354 3076

Email: oinzunza@med.puc.cl

Recibido : 13-01-2011

Aceptado: 26-04-2011 